\title{
Electromagnetic Modeling and Characterization of Steel Fiber Reinforced Concrete during the Pouring Process
}

\author{
G. Roqueta ${ }^{1}$, J. Romeu, and L. Jofre \\ AntennaLab, Universitat Politecnica de Catalunya (UPC), Spain \\ http://www.tsc.upc.edu/antennalab \\ ${ }^{1}$ gemma.roquetaetsc.upc.edu
}

\section{Introduction}

Thanks to its excellent mechanical properties, Steel Fiber Reinforced Concrete (SFRC) is being increasingly used in civil constructions. In order to ensure the prescribed tensile strength, the conglomerate needs to have a certain volumetric density of fibers (normally around $40 \mathrm{~kg} / \mathrm{m}^{3}$ ) uniformily distributed over the structure, and the inspection has to be done during the pouring process.

Up to the moment, no experiences have been reported concerning the variation of electrogmanetic properties of SFRC during the first stages after its fabrication. The resulting information of this study is crucial for the developement of a non-destructive inspection method for SFRC based on microwaves and previously overviewed in [1]. Microwaves, due to their capability to penetrate and sense light opaque materials with reasonable spatial resolution, can offer an interesting approach to the analysis of SFRC during the pouring process.

In this paper, a complex electromagnetical model for the SFRC is derived, and a mathematical model for the obtention of the effective relative permittivity is described. A static analysis setup is presented and results of a two weeks duration analysis are analyzed. Finally, some preliminary conclusions for the time-dependence behaviour of SFRC are formulated.

\section{Electromagnetic Modeling}

In electromagnetic theory, a metallic fiber can be considered as a prolate spheroid scatterer with length $l_{f i b}$ and diameter $d_{f i b}$, constituting an artificial dielectric. A randomly placed steel fiber induces a dipole moment accounting for the additional polarization density to host medium concrete. The average polarizability value for these metallic individual wires may be obtained as [2]:

$$
\alpha_{p}=\frac{\left(2 l_{f i b}\right)^{2}}{j \omega Z_{i n p}}
$$

where $Z_{i n p}$ is the input impedance of the wire antenna. Under the Maxwell - Garnett approach, the electric field inside the scatterers is determined assuming the diameter of the inclusion particles to be much smaller than the wavelength and considering that the distances between inclusions are large compared to the particle size. However, this model does not account for the mutual coupling between fibers. Mutual coupling may slightly decrease the ressonant frequency of the composite when the number of inclusions increases [3]. Under these circumstances, the well-known Clausius Mossotti formula

$$
\frac{\epsilon_{r e f f}-\epsilon_{r h}}{\epsilon_{r e f f}+2 \epsilon_{r h}}=\frac{n \alpha}{3 \epsilon_{r h} \epsilon_{0}}
$$


can be used to connect the relative effective permittivity $\epsilon_{\text {reff }}$ of a composite with the polarizability of the individual inclusion $\alpha_{p}$ and the number of inclusions in the unit volume $n . \epsilon_{r} h$ is the relative permittivity of the host medium and $\epsilon_{0}$ is the vacuum permittivity.

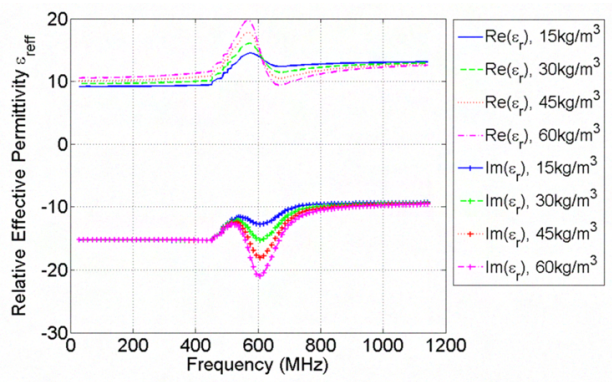

Figure 1: Real and Imaginary parts of $\epsilon_{\text {reff }}$ of PEC wires inside a concrete slab with $\epsilon_{r h}$ measured after $24 \mathrm{~h}$ of fabrication.

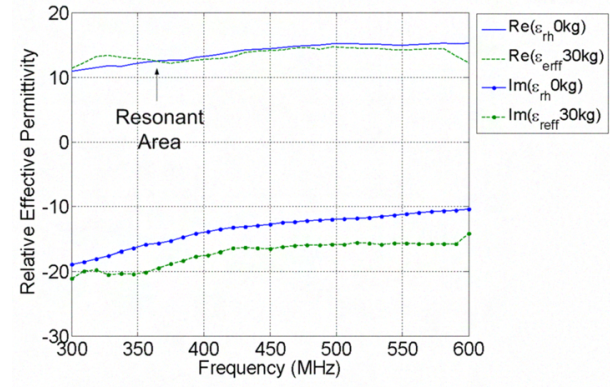

Figure 2: Real and Imaginary parts of the relative effective permittivity of the host medium ( $24 \mathrm{~h} 0 \mathrm{~kg} / \mathrm{m}^{3}$ concrete) $\epsilon_{r h}$ and $\epsilon_{\text {reff }}$ of a $24 \mathrm{~h} 30 \mathrm{~kg} / \mathrm{m}^{3}$ SFRC bloc.

Figure 1 represents the relative effective permittivity $\epsilon_{\text {reff }}$ of a composite material consisting on PEC wires $65 \mathrm{~mm}$ length and $0.5 \mathrm{~mm}$ diameter as a function of frequency in the UHF band $(300 \mathrm{MHz}-1.5 \mathrm{GHz})$, in a concrete host medium whose permittivity has been measured and is shown in Figure 2. The polarizability $\alpha$ has been computed using the NEC MoM code. Before the resonance $(550 \mathrm{MHz}), \operatorname{Re}\left(\epsilon_{\text {reff }}\right)$ increases proportionally to the density while after the resonance the behaviour of $\operatorname{Re}\left(\epsilon_{\text {reff }}\right)$ is the oposite. According to previous studies in [1], under-resonance regime is required for SFRC measurements.

\section{Experimental Determination of Complex Dielectric Constant}

Using the method described herein [4], the complex values of $\epsilon_{\text {rreff }}$ are determined from measurements of the scattering parameters. TRL calibration and measurements are obtained at the reference planes indicated in Figure 3. The effective relative permittivity of the material under test is related to the propagation constant through the Maxwell equations cited in [5]:

$$
\epsilon_{r e f f}=\frac{\beta^{2}-\alpha^{2}}{\omega^{2} \mu_{0} \epsilon_{0}}+j \frac{2 \beta \alpha}{\omega^{2} \mu_{0} \epsilon_{0}}
$$

where $\beta$ is the phase of the propagation vector known as $\beta=\frac{2 \Pi}{\lambda_{e f f}} d_{c o n c}$ and $\alpha$ is the attenuation of the propagation vector expressed as $e^{\left(-\alpha d_{c o n c}\right)}$. Scattering parameters are related to the transmission and reflection vector $\tau$ and $\rho$ through a two ports reflection and transmission diagram described in [4].

$$
\begin{aligned}
& S_{11}=S_{22}=\frac{V_{\text {out } 1}}{V_{\text {in }}}=\frac{\left(1-\tau^{2}\right) \rho}{1-\tau^{2} \rho^{2}} \\
& S_{21}=S_{12}=\frac{V_{\text {out } 2}}{V_{\text {in }}}=\frac{\left(1-\rho^{2}\right) \tau}{1-\tau^{2} \rho^{2}}
\end{aligned}
$$

where $V_{\text {in }}$ and $V_{\text {out } 1}$ are the input and the output of the system coming from port 1 and $V_{\text {out } 2}$ is the output of the system towards port 2. 


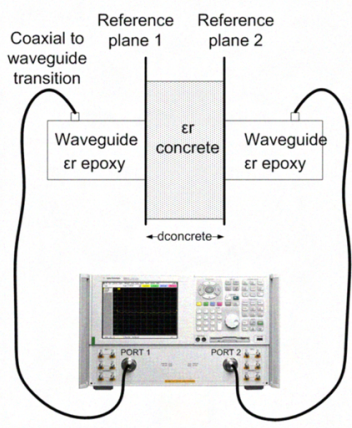

(a) Schematics

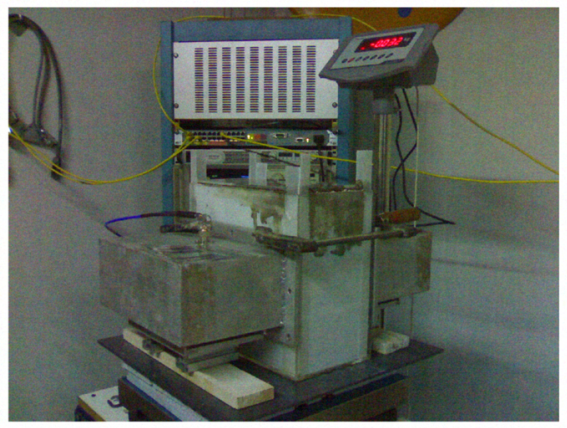

(b) Experimental Setup

Figure 3: Measurement setup for experimental determination of complex dielectric constant. (a) schematics and (b) experimental setup.

\section{Experimental Results}

In order to validate the previous model and to obtain practical data for the variation of dielectric properties of SFRC as a funtion of time and humidity content, Figure 3(a) has been built and different SFRC blocs $\left(0 \mathrm{~kg} / \mathrm{m}^{3}\right.$ and $\left.30 \mathrm{~kg} / \mathrm{m}^{3}\right)$ have been monitorized during two weeks. The equipment consists of two $300-600 \mathrm{MHz}$ [1] epoxy filled waveguides placed in a transmission arrangement propagating the illumination field through a $34 \mathrm{~cm} \times 30 \mathrm{~cm} \times 17 \mathrm{~cm}$ material sample and a high precision balance. Figure 2 shows how the retrieved relative permittivities for both blocs agree with the theoretical model described in this paper.

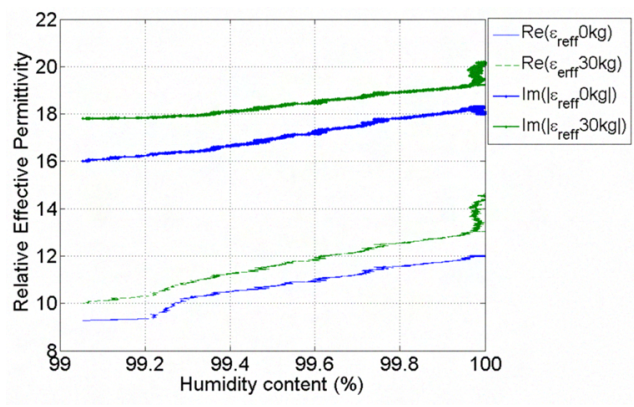

(a)

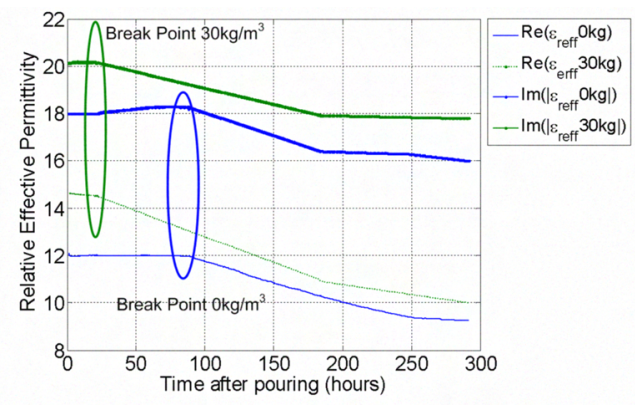

(b)

Figure 4: $\epsilon_{r e f f}$ dependence on (a) humidity content and (b) time.

Figure 3(b) shows the setup of the system. In a real pouring context the concrete slab is covered with a plastic cover during the first hours for a slower and more reliable compacting of the mixture. In the following experiences it will be shown that the effect of covering the slab or not is of great importance concerning the ressonant frequency, the water evaporation expressed on an apparent loss of weight, and variation of permittivity. Figure 4(a) and Figure 4(b) show the humidity content dependence and time dependence of the permittivity values for the $0 \mathrm{~kg} / \mathrm{m}^{3}$ and $30 \mathrm{~kg} / \mathrm{m}^{3}$ SFRC blocs. According to Figure 4(a), in both cases the loss of weight since the fabrication moment has approximately been of $1 \%$. 


\section{Conclusions}

An accurate study of the variation of dielectric properties of SFRC has been analitically developed and experimentally tested in a fifteen days measurement campaign. The system is able to discriminate between pouring process of $0 \mathrm{~kg} / \mathrm{m}^{3}$ and $30 \mathrm{~kg} / \mathrm{m}^{3}$, which conclusion could be extensible to other standard dosage values. The high humidity content of the composite produces a variation of the ressonant frequency towards the lowest values, which will conditionate the suitable frequency range for future measurements. Under the resonance, the measured permittivity increasing behaviour is compliant with the behaviour of $\epsilon_{\text {reff }}$ described in the electromagnetical model, although the resonance point has decreased due to coupling effects that are not being considered in the model.

Dielectric properties change in the order of three units with respect to the value in the initial stage of the pouring process. As observed in [6] for the pure concrete, it foreseen that $\epsilon_{r e f f^{\prime}}$

reaches a stable value about one month after concreting, but the stabilization of $\epsilon_{r e f f^{\prime \prime}}$ may need more than a month.

\section{Acknowledgments}

This project arises from the multidisciplinary group joining Signal Theory and Communications department, and Civil Engineering department, headed by Professor Antonio Aguado, to whom special thanks are addressed. Thanks also to Professor J. M. Torrents for his support on the conception of the measurement and procurement of the SFRC samples. This work was supported in part by the Spanish Interministerial Commission on Science and Technology (CICYT) under projects TEC2007-66698-C04-01/TCM and CONSOLIDER CSD2008-00068 and by the "Ministerio de Educacin y Ciencia" through the FPU fellowship program.

\section{References}

[1] G. Roqueta, B. Monsalve, S. Blanch, J. Romeu, and L. Jofre, "Microwave dielectric properties inspection of fiber-reinforced civil structures," in Proc. IEEE Antennas and Propagation Society International Symposium AP-S 2008, 5-11 July 2008, pp. 1-4.

[2] S. A. Tretyakov, S. Maslovski, and P. A. Belov, "An analytical model of metamaterials based on loaded wire dipoles," vol. 51, no. 10, pp. 2652-2658, Oct. 2003.

[3] C. Manolatou, "Calculation of effective electromagnetic parametres of helic loaded composites," Master's thesis, Massachusetts Insitute of Technology, 1995.

[4] W. B. Weir, "Automatic measurement of complex dielectric constant and permeability at microwave frequencies," vol. 62, no. 1, pp. 33-36, Jan. 1974.

[5] C. A. Balanis, Advanced Engineering Electromagnetics, J. W. . Sons, Ed., 1989.

[6] K. J. Bois, A. D. Benally, and R. Zoughi, "Microwave near-field reflection property analysis of concrete for material content determination," vol. 49, no. 1, pp. 49-55, Feb. 2000 . 